\title{
Generalized Convergence Rates Results for Linear Inverse Problems in Hilbert Spaces
}

\author{
Roman Andreev* Peter Elbau ${ }^{\dagger} \quad$ Maarten V. de Hoop ${ }^{\ddagger}$ \\ Lingyun $\mathrm{Qiu}^{\S} \quad$ Otmar Scherzer $₫$
}

September 19, 2018

\begin{abstract}
In recent years, a series of convergence rates conditions for regularization methods has been developed. Mainly, the motivations for developing novel conditions came from the desire to carry over convergence rates results from the Hilbert space setting to generalized Tikhonov regularization in Banach spaces. For instance, variational source conditions have been developed and they were expected to be equivalent to standard source conditions for linear inverse problems in a Hilbert space setting (see Schuster et al [10]). We show that this expectation does not hold. However, in the standard Hilbert space setting these novel conditions are optimal, which we prove by using some deep results from Neubauer [8], and generalize existing convergence rates results. The key tool in our analysis is a novel source condition, which we put into relation to the existing source conditions from the literature. As a positive by-product, convergence rates results can be proven without spectral theory, which is the standard technique for proving convergence rates for linear inverse problems in Hilbert spaces (see Groetsch [4]).
\end{abstract}

\section{Introduction}

In this paper we consider for some (not exactly known) data $y \in \mathcal{R}(L)$ the operator equation

$$
L u=y,
$$

*Johann Radon Institute for Computational and Applied Mathematics (RICAM), Altenbergerstrasse 69, A-4040 Linz, Austria (roman.andreev@oeaw.ac.at)

${ }^{\dagger}$ Computational Science Center, University of Vienna, Oskar-Morgenstern Platz 1, A-1090 Vienna, Austria (peter.elbau@univie.ac.at)

$\ddagger$ Center for Computational and Applied Mathemematics, Purdue University, West Lafayette, IN 47907, USA (mdehoop@purdue.edu)

$\S$ Institute for Mathematics and its Applications, University of Minnesota, Minneapolis, MN 55455, USA (qiu.lingyun@ima.umn.edu)

TComputational Science Center, University of Vienna and RICAM (otmar.scherzer@univie.ac.at) 
where $L: U \rightarrow V$ is a bounded linear operator between two real Hilbert spaces $U$ and $V$, and $\mathcal{R}(L)$ denotes its range.

Given some approximate data $y^{\delta} \in V$ with $\left\|y-y^{\delta}\right\| \leq \delta$, the objective is to reconstruct the minimal norm solution $u^{\dagger} \in U$, that is the element fulfilling

$$
L u^{\dagger}=y \quad \text { and } \quad\left\|u^{\dagger}\right\|=\inf \{\|u\|: L u=y\} .
$$

Such a minimal norm solution exists for every $y \in \mathcal{R}(L)$ and is uniquely defined, see for example [3, Theorem 2.5].

The method of choice for performing this task is Tikhonov regularization, that is to find for arbitrary $\alpha>0$ the regularized solution

$$
u_{\alpha}^{\delta}:=\underset{u \in U}{\operatorname{argmin}}\left\{\left\|L u-y^{\delta}\right\|^{2}+\alpha\|u\|^{2}\right\} .
$$

Standard results on Tikhonov regularization guarantee the existence and uniqueness of the minimizer $u_{\alpha}^{\delta}$ and that $u_{\alpha}^{\delta}$ converges to $u^{\dagger}$ for an appropriate choice of $\alpha$ depending on $\delta$ as $\delta \searrow 0$, see for instance [3, Theorem 5.1 and Theorem 5.2].

Convergence rates conditions, moreover, guarantee a certain convergence rate $\left\|u_{\alpha}^{\delta}-u^{\dagger}\right\|=\mathcal{O}(f(\delta))$ as $\delta \searrow 0$ if again $\alpha$ is chosen to depend in the right way on $\delta$.

Two kinds of such convergence rates conditions have been developed:

- source conditions [4] and

- variational source conditions $[6,9,5,10]$.

The goal of this paper is to put the different source conditions into perspective, together with three novel variational source conditions, which are presented here for the first time. The main results on the relations in between the source conditions are summarized in a table form (cf. Figure 1). Aside from these particular conditions the novelties are to show that these conditions are in fact more general than the classical source conditions, and they are optimal in the sense that convergence rates of a certain order are only possible if these conditions are satisfied. The argumentation is based on a result from Neubauer [8]. Moreover, as a side product, this clarifies some assertion from [10] on the equivalence of standard and variational source conditions.

\section{Relations of Source Conditions in the Stan- dard Setting}

The key to obtain convergence rate results for the regularized solution $u_{\alpha}^{\delta}$, defined in (2), of the problem (1) is to impose conditions on the minimal norm solution $u^{\dagger}$. In the literature, various kind of such source conditions have been introduced. 
Definition 1. Let $U$ and $V$ be real Hilbert spaces, $L: U \rightarrow V$ be a bounded linear operator, and $y \in \mathcal{R}(L)$. Moreover, let $u^{\dagger}$ denote the minimum-norm solution of the operator equation (1).

Then, we say that the problem fulfills

- the standard source condition, see [4], with the parameter $\nu \in(0,2]$ if

$$
u^{\dagger} \in \mathcal{R}\left(\left(L^{*} L\right)^{\frac{\nu}{2}}\right)
$$

- the homogeneous variational inequality with the parameter $\nu \in(0,1]$ if there exists a constant $\beta \geq 0$ such that

$$
2\left\langle u^{\dagger}, u\right\rangle \leq \beta\|L u\|^{\nu}\|u\|^{1-\nu} \quad \text { for every } \quad u \in U ;
$$

- the inhomogeneous variational inequality with the parameter $\mu \in(0,1]$, introduced in [6] for the case $\mu=1$ and in [5] for general $\mu \in(0,1]$ in the setting of non-linear problems, if there exist constants $\beta \geq 0$ and $\gamma \in[0,1)$ such that

$$
2\left\langle u^{\dagger}, u\right\rangle \leq \beta\|L u\|^{\mu}+\gamma\|u\|^{2} \quad \text { for every } \quad u \in U ;
$$

- the symmetrized variational inequality with the parameter $\nu \in(0,2]$ if there exists a constant $\beta \geq 0$ such that

$$
2\left\langle u^{\dagger}, u\right\rangle \leq \beta\left\|L^{*} L u\right\|^{\frac{\nu}{2}}\|u\|^{1-\frac{\nu}{2}} \quad \text { for every } \quad u \in U .
$$

Remark: Let $\rho \in(0,2]$. The family of variational source conditions,

$$
2\left\langle u^{\dagger}, u\right\rangle \leq \beta\left\|\left(L^{*} L\right)^{\frac{\rho}{2}} u\right\|^{\frac{\nu}{\rho}}\|u\|^{1-\frac{\nu}{\rho}}, \quad \nu \leq \rho,
$$

puts the homogeneous variational inequality, the symmetrized variational inequality, and the standard source condition under one umbrella, when we set $\rho=1, \rho=2$, and $\rho=\nu$ (see the proof of Lemma 3(ii)), respectively. However, Proposition 11 and Proposition 10 show that all these variational source conditions with the same parameter $\nu$ and a parameter $\rho>\nu$ are equivalent to each other.

Note that the inhomogeneous variational inequality is not homogeneous with respect to $u \in U$, as opposed to the other three source conditions.

Let us first discuss the relation between the first three source conditions.

Lemma 2. Let $U$ and $V$ be real Hilbert spaces, $L: U \rightarrow V$ be a bounded linear operator, $y \in \mathcal{R}(L)$, and $\nu \in(0,1]$. Then, we have that

(i) the standard source condition for $\nu$ implies the homogeneous variational inequality with the same parameter $\nu$,

(ii) the homogeneous variational inequality with the parameter $\nu$ implies the inhomogeneous variational inequality with the parameter $\mu=\frac{2 \nu}{1+\nu}$, and 
(iii) the inhomogeneous variational inequality with the parameter $\mu=1$ implies the standard source condition with the parameter $\nu=1$.

Proof: Let $u^{\dagger}$ denote the minimum-norm solution of the operator equation (1).

(i) If the standard source condition is fulfilled for some $\nu \in(0,1]$, then there exists an element $\omega \in U$ with $\left(L^{*} L\right)^{\frac{\nu}{2}} \omega=u^{\dagger}$. Using now the interpolation inequality

$$
\left\|\left(L^{*} L\right)^{r} u\right\| \leq\left\|\left(L^{*} L\right)^{q} u\right\|^{\frac{r}{q}}\|u\|^{1-\frac{r}{q}} \quad \text { for all } \quad u \in U, 0<r \leq q,
$$

see for example [3, Chapter 2.3], with $r=\frac{\nu}{2}$ and $q=\frac{1}{2}$, it follows for every $u \in U$ that

$$
2\left\langle u^{\dagger}, u\right\rangle=\left\langle 2 \omega,\left(L^{*} L\right)^{\frac{\nu}{2}} u\right\rangle \leq 2\|\omega\|\left\|\left(L^{*} L\right)^{\frac{\nu}{2}} u\right\| \leq 2\|\omega\|\|L u\|^{\nu}\|u\|^{1-\nu},
$$

which is of the form (4) with the parameter $\nu$.

(ii) If $u^{\dagger}$ fulfills the variational inequality (4) for some parameters $\nu \in(0,1]$ and $\beta \geq 0$, then Young's inequality implies for every $u \in U$ that

$$
2\left\langle u^{\dagger}, u\right\rangle \leq \beta\|L u\|^{\nu}\|u\|^{1-\nu} \leq \frac{1+\nu}{2} \beta^{\frac{2}{1+\nu}}\|L u\|^{\frac{2 \nu}{1+\nu}}+\frac{1-\nu}{2}\|u\|^{2},
$$

so that the inhomogeneous variational inequality with the parameter $\mu=$ $\frac{2 \nu}{1+\nu}$ is fulfilled.

(iii) If $u^{\dagger}$ fulfills the inequality (5) for $\mu=1$ and some constants $\beta \geq 0$ and $\gamma \in[0,1)$, then, by evaluating it at $u=t v$ for arbitrary $v \in U$ and $t>0$, we find in the limit $t \searrow 0$ that

$$
2\left\langle u^{\dagger}, v\right\rangle \leq \beta\|L v\|=\beta\left\|\left(L^{*} L\right)^{\frac{1}{2}} v\right\| \quad \text { for every } \quad v \in U .
$$

Now, it can be shown, see [9, Lemma 8.21], that if $T: U \rightarrow U$ is a bounded linear operator, then $u^{\dagger} \in \mathcal{R}\left(T^{*}\right)$ if and only if there exists a constant $C>0$ such that $\left\langle u^{\dagger}, v\right\rangle \leq C\|T v\|$ for all $v \in U$.

Thus, with $T=\left(L^{*} L\right)^{\frac{1}{2}}$, we find that (8) is equivalent to $u^{\dagger} \in \mathcal{R}\left(\left(L^{*} L\right)^{\frac{1}{2}}\right)$.

Remark: That the standard source condition for a parameter $\nu \in(0,1]$ implies the inhomogeneous variational inequality with the parameter $\mu=\frac{2 \nu}{1+\nu}$ was already realized in [7].

The case $\nu=1$ has been treated in more generality in [9, Table 3.1].

Thus, the homogeneous variational inequality and the inhomogeneous variational inequality cover only the parameter range $\nu \in(0,1]$ compared to the standard source condition. However, the symmetrized variational inequality is an extension of the standard source condition in the full parameter range $\nu \in(0,2]$, as the following lemma shows. 
Lemma 3. Let $U$ and $V$ be real Hilbert spaces, $L: U \rightarrow V$ be a bounded linear operator, and $y \in \mathcal{R}(L)$.

Then, we have that

(i) the standard source condition with a parameter $\nu \in(0,2]$ implies the symmetrized variational inequality with the same parameter $\nu$,

(ii) the symmetrized variational inequality with the parameter $\nu=2$ is equivalent to the standard source condition with the parameter $\nu=2$.

Proof: Let $u^{\dagger}$ denote the minimum-norm solution of the problem (1).

(i) From the inequality (6) with some parameters $\nu \in(0,1]$ and $\beta \geq 0$, we obtain by applying the Cauchy-Schwarz inequality for every $u \in U$ that

$$
2\left\langle u^{\dagger}, u\right\rangle \leq \beta\left\langle L^{*} L u, u\right\rangle^{\frac{\nu}{2}}\|u\|^{1-\nu} \leq \beta\left\|L^{*} L u\right\|^{\frac{\nu}{2}}\|u\|^{1-\frac{\nu}{2}},
$$

which is the symmetrized variational inequality with the parameter $\nu$.

(ii) The symmetrized variational inequality with the parameter $\nu=2$ states that there exists a constant $\beta \geq 0$ so that

$$
\left\langle u^{\dagger}, u\right\rangle \leq \beta\left\|L^{*} L u\right\| \quad \text { for all } u \in U .
$$

Now, as in the proof of Lemma 2(iii), this is equivalent to $u^{\dagger} \in \mathcal{R}\left(L^{*} L\right)$, see [9, Lemma 8.21].

The following two examples illustrate that the degree of ill-posedness of the operator $L$ is a criterion for equivalency of the different source conditions. Finer results, establishing in particular the equivalence of the source conditions (4) and (5) and the corresponding convergence rates, see Proposition 8, will be derived in Theorem 12 below.

Example 4. Let $U$ and $V$ be real Hilbert spaces, $\nu \in(0,1], L: U \rightarrow V$ be a bounded linear operator so that $\left(L^{*} L\right)^{\nu / 2}$ has closed range, and $y \in \mathcal{R}(L)$.

Then,

(i) the standard source condition with parameter $\nu$,

(ii) the homogeneous variational inequality with parameter $\nu$, and

(iii) the inhomogeneous variational inequality with parameter $\mu=\frac{2 \nu}{1+\nu}$

are equivalent.

Proof: In view of Lemma 2, we only need to show that (iii) implies (i). To that end recall that, if $T: U \rightarrow U$ is a bounded linear self-adjoint operator then its nullspace $\mathcal{N}(T)$ is the orthogonal complement of the range $\mathcal{R}(T)$, and $U=\overline{\mathcal{R}(T)} \oplus \mathcal{N}(T)$. Since the range of $T=\left(L^{*} L\right)^{\nu / 2}$ is closed by assumption, we have the orthogonal decomposition

$$
U=\mathcal{R}\left(\left(L^{*} L\right)^{\frac{\nu}{2}}\right) \oplus \mathcal{N}\left(\left(L^{*} L\right)^{\frac{\nu}{2}}\right) .
$$


Observe now that, if $u \in \mathcal{N}\left(\left(L^{*} L\right)^{\frac{\nu}{2}}\right)$ then

$$
\|L u\|^{2}=\langle L u, L u\rangle=\left\langle\left(L^{*} L\right)^{\nu / 2} u,\left(L^{*} L\right)^{1-\nu / 2} u\right\rangle=0,
$$

so that $L u=0$. Therefore, if $u^{\dagger}$ satisfies (5) with some constants $\beta \geq 0$ and $\gamma \in[0,1)$, then

$$
2\left\langle u^{\dagger}, u\right\rangle \leq \gamma\|u\|^{2} \quad \text { for every } \quad u \in \mathcal{N}\left(\left(L^{*} L\right)^{\nu / 2}\right) .
$$

Substituting $u$ by $t u$ in the above inequality with $t>0$, we arrive at

$$
2 t\left\langle u^{\dagger}, u\right\rangle \leq t^{2} \gamma\|u\|^{2} \quad \text { for every } \quad u \in \mathcal{N}\left(\left(L^{*} L\right)^{\nu / 2}\right), t>0 .
$$

Dividing by $t$ and letting $t$ go to 0 , this implies $\left\langle u^{\dagger}, u\right\rangle=0$ whenever $u \in$ $\mathcal{N}\left(\left(L^{*} L\right)^{\nu / 2}\right)$. By the orthogonality of the decomposition (9) we have $u^{\dagger} \in$ $\mathcal{R}\left(\left(L^{*} L\right)^{\nu / 2}\right)$, which is (i).

Remark: As in Example 4, one can also show that the standard source condition and the symmetrized variational inequality with the same parameter $\nu \in(0,2]$ are equivalent if $\left(L^{*} L\right)^{\frac{\nu}{2}}$ has closed range.

Example 5. Let $U$ be a real, separable Hilbert space with orthonormal basis $\left\{\varphi_{n}\right\}_{n \in \mathbb{N}}$. We define the compact linear operator $L: U \rightarrow U$ by $L\left(\varphi_{n}\right)=2^{-n} \varphi_{n}$. (Note that its range is not closed, for the range of a compact operator is closed if and only if it is finite-dimensional.)

Then, for the data

$$
y=\sum_{n \geq 1} 2^{-\frac{3}{2} n} \varphi_{n} \in \mathcal{R}(L),
$$

the problem (1) fulfills the homogeneous variational inequality with the parameter $\nu=\frac{1}{2}$, but not the standard source condition with parameter $\nu=\frac{1}{2}$. In particular, the two source conditions are not equivalent.

However, the standard source condition is fulfilled for every parameter $\nu<\frac{1}{2}$.

Proof: The minimum-norm solution $u^{\dagger}$ can be directly calculated to be

$$
u^{\dagger}=L^{-1} y=\sum_{n \geq 1} 2^{-\frac{n}{2}} \varphi_{n} .
$$

Now, since $L$ is self-adjoint by definition so that we have $\left(L^{*} L\right)^{\frac{1}{2}}=L$, we see that $u^{\dagger} \notin \mathcal{R}\left(\left(L^{*} L\right)^{\frac{1}{4}}\right)$ because $L^{-\frac{1}{2}} u^{\dagger}=\sum_{n \geq 1} \varphi_{n} \notin U$.

However, we have for every $\nu<\frac{1}{2}$ that $L^{-\nu} u^{\dagger}=\sum_{n \geq 1} 2^{n\left(\nu-\frac{1}{2}\right)} \varphi_{n}$ is in $U$, and therefore $u^{\dagger}$ is in the range of $\left(L^{*} L\right)^{\frac{\nu}{2}}$ for every $\nu<\frac{1}{2}$.

For $u \in U$ arbitrary we write $u=\sum_{n \geq 1} 2^{\frac{n}{2}} \gamma_{n} \varphi_{n}$ with some $\gamma_{n} \in \mathbb{R}$. Then

$$
\left\langle u^{\dagger}, u\right\rangle=\sum_{n \geq 1} \gamma_{n}, \quad\|u\|^{2}=\sum_{n \geq 1} 2^{n}\left|\gamma_{n}\right|^{2}, \quad \text { and } \quad\|L u\|^{2}=\sum_{n \geq 1} 2^{-n}\left|\gamma_{n}\right|^{2} .
$$


Now we can show that the homogeneous variational inequality with parameter $\nu=\frac{1}{2}$ is fulfilled, more precisely, that we have

$$
\left\langle u^{\dagger}, u\right\rangle \leq 2 \sqrt{2}\|u\|^{\frac{1}{2}}\|L u\|^{\frac{1}{2}} \quad \text { for every } \quad u \in U .
$$

Indeed, set $S:=\sum_{n \geq 1}\left|\gamma_{n}\right|$ and let $N \in \mathbb{N}$ be such that

$$
\frac{1}{2} S \leq A:=\sum_{n \leq N}\left|\gamma_{n}\right| \quad \text { and } \quad \frac{1}{2} S \leq B:=\sum_{n \geq N}\left|\gamma_{n}\right| .
$$

Observe that, using the Cauchy-Schwarz inequality,

$$
A^{2} \leq \sum_{k \leq N} 2^{k} \sum_{n \leq N} 2^{-n}\left|\gamma_{n}\right|^{2} \leq\left(2^{N+1}-1\right)\|L u\|^{2},
$$

and

$$
B^{2} \leq \sum_{k \geq N} 2^{-k} \sum_{n \geq N} 2^{n}\left|\gamma_{n}\right|^{2} \leq 2^{-N+1}\|u\|^{2}
$$

Since we have by definition of $S$ that $\left\langle u^{\dagger}, u\right\rangle \leq S$ and by the choice of $N$ that $S \leq 2 \sqrt{A B}$, the inequality (11) follows.

This proof is largely from [11]. The proof of Proposition 11 below is a more elaborate version of the same idea.

Remark: In the above proof we noted that

$$
u^{\dagger} \in \mathcal{R}\left(\left(L^{*} L\right)^{\frac{\rho}{2}}\right) \quad \text { for every } \quad \rho \in[0, \nu) .
$$

This property is a general consequence of the variational source condition (4). This follows from Proposition 8 and [8, Corollary 2.4].

However, if $u^{\dagger}$ satisfies (12), it need not satisfy (4): take $u^{\dagger}$ as in (10) but with $L\left(\varphi_{n}\right):=n^{-2} 2^{-n} \varphi_{n}$. Then (12) holds for $\nu=1 / 2$. But $\left\langle u^{\dagger}, \varphi_{n}\right\rangle=2^{-n / 2}$ is not bounded in terms of $\left\|L \varphi_{n}\right\|^{1 / 2}\left\|\varphi_{n}\right\|^{1 / 2}=n^{-1} 2^{-n / 2}$ uniformly in $n \geq 1$.

\section{Rates Results without Spectral Theory}

We briefly review the convergence rate results which follow from the introduced source conditions.

Definition 6. Let $U$ and $V$ be real Hilbert spaces, $L: U \rightarrow V$ be a bounded linear operator, and $y \in \mathcal{R}(L)$. Moreover, let $u^{\dagger}$ denote the minimum-norm solution of the operator equation (1).

Then, we say that the problem has

- a noise-free convergence rate of order $\sigma$ if there exists a constant $C>0$ so that the regularized solution

$$
u_{\alpha}=\underset{u \in U}{\operatorname{argmin}}\left(\|L u-y\|^{2}+\alpha\|u\|^{2}\right),
$$

fulfills that

$$
\left\|u_{\alpha}-u^{\dagger}\right\| \leq C \alpha^{\sigma} \quad \text { for every } \quad \alpha>0,
$$


- a convergence rate of order $\rho$ if there exists a constant $C>0$ so that the regularized solutions

$$
u_{\alpha}(\tilde{y})=\underset{u \in U}{\operatorname{argmin}}\left(\|L u-\tilde{y}\|^{2}+\alpha\|u\|^{2}\right), \quad \alpha>0, \tilde{y} \in V,
$$

fulfill for every $\delta>0$ the inequality

$$
\sup \left\{\inf _{\alpha>0}\left\|u_{\alpha}(\tilde{y})-u^{\dagger}\right\|: \tilde{y} \in V,\|\tilde{y}-y\| \leq \delta\right\} \leq C \delta^{\rho} .
$$

The classical convergence results now state that if a problem (1) fulfils the standard source condition for some parameter $\nu \in(0,2]$, then it has a convergence rate of order $\frac{\nu}{1+\nu}$, see [4, Corollary 3.1.4]. For $\nu, \mu \in(0,1]$ the same result can be obtained under the weaker source conditions (4) and $(5)$, see $[5,10]$. The simple proof is added here for completeness.

Lemma 7. Let $L: U \rightarrow V$ be a bounded linear operator between two real Hilbert spaces $U$ and $V$, and $y \in \mathcal{R}(L)$. Moreover, let $u^{\dagger}$ denote the minimum-norm solution of the problem (1) and assume that it fulfils the inhomogeneous variational inequality (5) for some parameters $\mu \in(0,1], \beta \geq 0$, and $\gamma \in(0,1)$.

Then, for every choice of $y^{\delta} \in V$ with $\left\|y^{\delta}-y\right\| \leq \delta$ for some $\delta>0$ and every $\alpha>0$, the corresponding regularized solution

$$
u_{\alpha}^{\delta}=\underset{u \in U}{\operatorname{argmin}}\left(\left\|L u-y^{\delta}\right\|^{2}+\alpha\|u\|^{2}\right)
$$

satisfies

$$
\left\|u_{\alpha}^{\delta}-u^{\dagger}\right\|^{2} \leq \frac{2}{1-\gamma} \frac{\delta^{2}}{\alpha}+\frac{\beta^{\frac{2}{2-\mu}}(2-\mu)}{2(1-\gamma)} \alpha^{\frac{\mu}{2-\mu}}
$$

Proof: From the definition of the minimizer $u_{\alpha}^{\delta}$, it follows that

$$
\left\|L u_{\alpha}^{\delta}-y^{\delta}\right\|^{2}+\alpha\left\|u_{\alpha}^{\delta}\right\|^{2} \leq \delta^{2}+\alpha\left\|u^{\dagger}\right\|^{2} .
$$

This inequality together with the variation inequality (5) yields

$$
\begin{aligned}
\left\|L u_{\alpha}^{\delta}-y^{\delta}\right\|^{2}+\alpha\left\|u_{\alpha}^{\delta}-u^{\dagger}\right\|^{2} & \leq \delta^{2}+2 \alpha\left\langle u^{\dagger}, u^{\dagger}-u_{\alpha}^{\delta}\right\rangle \\
& \leq \delta^{2}+\alpha \beta\left\|L\left(u_{\alpha}^{\delta}-u^{\dagger}\right)\right\|^{\mu}+\alpha \gamma\left\|u_{\alpha}^{\delta}-u^{\dagger}\right\|^{2} .
\end{aligned}
$$

Now, observing that

$$
\frac{1}{2}\left\|L\left(u_{\alpha}^{\delta}-u^{\dagger}\right)\right\|^{2}-\delta^{2} \leq\left\|L u_{\alpha}^{\delta}-y^{\delta}\right\|^{2},
$$

which is a consequence of the triangle inequality and the fact that $a \leq b+c$ implies $a^{2} \leq 2\left(b^{2}+c^{2}\right)$, we further find that

$$
\frac{1}{2}\left\|L\left(u_{\alpha}^{\delta}-u^{\dagger}\right)\right\|^{2}+\alpha(1-\gamma)\left\|u_{\alpha}^{\delta}-u^{\dagger}\right\|^{2} \leq 2 \delta^{2}+\alpha \beta\left\|L\left(u_{\alpha}^{\delta}-u^{\dagger}\right)\right\|^{\mu} .
$$


Applying then Young's inequality to the last term, we end up with

$\frac{1}{2}\left\|L\left(u_{\alpha}^{\delta}-u^{\dagger}\right)\right\|^{2}+\alpha(1-\gamma)\left\|u_{\alpha}^{\delta}-u^{\dagger}\right\|^{2} \leq 2 \delta^{2}+\frac{2-\mu}{2}(\alpha \beta)^{\frac{2}{2-\mu}}+\frac{\mu}{2}\left\|L\left(u_{\alpha}^{\delta}-u^{\dagger}\right)\right\|^{2}$,

which in particular implies (16).

Proposition 8. Assume that $L: U \rightarrow V$ is a bounded linear operator between two real Hilbert spaces $U$ and $V$, and $y \in \mathcal{R}(L)$.

Then, if the problem (1) fulfills the inhomogeneous variational inequality with the parameter $\mu=\frac{2 \nu}{1+\nu}$ for some $\nu \in(0,1]$, it has

(i) a noise-free convergence rate of order $\frac{\nu}{2}$ and

(ii) a convergence rate of order $\frac{\nu}{1+\nu}$.

Proof: Let $u^{\dagger}$ be the minimal-norm solution of (1).

(i) In the noise free case, Lemma 7 with $\delta=0$ and $\mu=\frac{2 \nu}{1+\nu}$ directly implies for the regularized solution $u_{\alpha}$ defined by (13) the inequality

$$
\left\|u_{\alpha}-u^{\dagger}\right\| \leq C \alpha^{\frac{\mu}{2(2-\mu)}}=C \alpha^{\frac{\nu}{2}} \text { for all } \quad \alpha>0
$$

for some constant $C>0$.

(ii) In the noisy case, Lemma 7 yields for arbitrary $\delta>0$ and data $\tilde{y} \in V$ with $\|\tilde{y}-y\| \leq \delta$ the inequality

$$
\inf _{\alpha>0}\left\|u_{\alpha}(\tilde{y})-u^{\dagger}\right\|^{2} \leq\left\|u_{\delta^{2-\mu}}(\tilde{y})-u^{\dagger}\right\|^{2} \leq C \delta^{\mu}=C \delta^{\frac{2 \nu}{1+\nu}}
$$

for some constant $C>0$. Here, $u_{\alpha}(\tilde{y})$ denotes the regularized solution (14).

Remark: Because of Lemma 2, the homogeneous variational inequality with a parameter $\nu \in(0,1]$ therefore also implies a noise-free convergence rate of order $\frac{\nu}{2}$ and a convergence rate of order $\frac{\nu}{1+\nu}$.

\section{On converse results of Neubauer}

In this section we go deeper into the results of Neubauer [8]. In the Hilbert space setting, Neubauer characterized the minimum-norm solution for which the problem has a convergence rate of order $\frac{\nu}{\nu+1}$ for some $\nu \in(0,2)$ in terms of its spectral tail. (Note that Neubauer writes $2 \nu$ where we write $\nu$.)

Definition 9. Let $U$ and $V$ be real Hilbert spaces, $L: U \rightarrow V$ be a bounded linear operator, and $y \in \mathcal{R}(L)$. We say that the minimum-norm solution $u^{\dagger}$ of the problem (1) has spectral tail of order $\nu$ if there exists a constant $C>0$ so that

$$
\left\|E_{[0, \lambda]} u^{\dagger}\right\|^{2} \leq C^{2} \lambda^{\nu} \quad \text { for all } \quad \lambda \geq 0
$$

where $A \mapsto E_{A}$ denotes the (projection-valued) spectral measure of the operator $L^{*} L$. 
Proposition 10. Let $L: U \rightarrow V$ be a bounded linear operator between two real Hilbert spaces $U$ and $V$, and $y \in \mathcal{R}(L)$.

Then, for every $\nu \in(0,2)$, it is equivalent for the problem (1) that

(i) it has a noise-free convergence rate of order $\frac{\nu}{2}$,

(ii) it has a convergence rate of order $\frac{\nu}{\nu+1}$, and

(iii) its minimum-norm solution has a spectral tail of order $\nu$.

Proof: Neubauer showed in [8, Theorem 2.1] that the condition (i) is equivalent to (iii), and proved in [8, Theorem 2.6] that (iii) is equivalent to the fact that there exists a constant $C \geq 0$ so that

$$
\sup \left\{\inf _{\alpha>0}\left\|u_{\alpha}(\tilde{y})-u^{\dagger}\right\|: \tilde{y} \in V,\|Q(\tilde{y}-y)\| \leq \delta\right\} \leq C \delta^{\frac{\nu}{\nu+1}}
$$

for every $\delta \geq 0$, where $Q$ denotes the orthogonal projection onto the range $\overline{\mathcal{R}(L)}$ and the regularized solution $u_{\alpha}(\tilde{y})$ is defined by (14).

It therefore only remains to show that (19) is equivalent to a convergence rate of order $\frac{\nu}{\nu+1}$.

It is clear that (19) implies such a convergence rate, since the supremum in the definition (15) of the convergence rate is taken over a smaller set than in (19).

For the other direction, we define for arbitrary $\tilde{y} \in V$ with $\|Q(\tilde{y}-y)\| \leq \delta$, the element $\hat{y}:=y+Q(\tilde{y}-y)$. Then,

$$
\|\hat{y}-y\|=\|Q(\tilde{y}-y)\| \leq \delta,
$$

and the optimality conditions for the regularized solutions $u_{\alpha}(\tilde{y})$ and $u_{\alpha}(\hat{y})$ yield

$$
u_{\alpha}(\tilde{y})-u_{\alpha}(\hat{y})=\left(\alpha I+L^{*} L\right)^{-1} L^{*}(\tilde{y}-\hat{y}) .
$$

Since now $L^{*}=L^{*} Q$, we have $L^{*}(\tilde{y}-\hat{y})=L^{*} Q(\tilde{y}-\hat{y})=0$ and therefore, $u_{\alpha}(\tilde{y})=u_{\alpha}(\hat{y})$. As $\tilde{y}$ was arbitrary subject to $\|Q(\tilde{y}-y)\| \leq \delta$, condition (15) with $\rho=\frac{\nu}{\nu+1}$ implies (19).

Neubauer [8] also gave a counterexample to show that the standard source condition with parameter $\nu \in(0,2)$, which implies the three equivalent conditions of Proposition 10, is not equivalent to them, see also Example 5.

However, we will show in the following that the homogeneous variational inequality with parameter $\nu \in(0,1)$ and the inhomogeneous variational inequality with parameter $\mu=\frac{2 \nu}{1+\nu}$ are indeed equivalent to the conditions of Proposition 10.

Proposition 11. Let $L: U \rightarrow V$ be a bounded linear operator between two real Hilbert spaces $U$ and $V$, and let $y \in \mathcal{R}(L)$.

Then for arbitrary $\nu \in(0,2)$ and $\rho>\nu$ the conditions that 
(i) the maximum-norm solution $u^{\dagger}$ of the problem (1) has a spectral tail of order $\nu$ and

(ii) there exists a constant $\beta \geq 0$ so that

$$
2\left\langle u^{\dagger}, u\right\rangle \leq \beta\left\|\left(L^{*} L\right)^{\frac{\rho}{2}} u\right\|^{\frac{\nu}{\rho}}\|u\|^{1-\frac{\nu}{\rho}} \quad \text { for all } \quad u \in U
$$

are equivalent.

Proof: We first show that (i) implies (ii).

Let $A \mapsto E_{A}$ denote the (projection-valued) spectral measure of $L^{*} L$. For arbitrary $u \in U$, we define the signed measure $A \mapsto \mu_{u^{\dagger}, u}(A)=\left\langle E_{A} u^{\dagger}, u\right\rangle$ and set for $\lambda \in[0, \infty]$

$$
A_{\lambda}:=\left|\mu_{u^{\dagger}, u}\right|([0, \lambda]) \quad \text { and } \quad B_{\lambda}:=\left|\mu_{u^{\dagger}, u}\right|([\lambda, \infty)),
$$

where $\left|\mu_{u^{\dagger}, u}\right|$ denotes the variation of the measure $\mu_{u^{\dagger}, u}$.

Let now $\Lambda:=\inf \left\{\lambda \geq 0: A_{\lambda} \geq \frac{1}{2} A_{\infty}\right\}$. Then, since $\lambda \mapsto A_{\lambda}$ is rightcontinuous, there holds $A_{\Lambda} \geq \frac{1}{2} A_{\infty}$. Moreover, since $\Lambda$ is minimal and $\lambda \mapsto B_{\lambda}$ is left-continuous, it also follows that $B_{\Lambda} \geq \frac{1}{2} A_{\infty}$.

We now estimate $A_{\Lambda}$ with the inequality (23) with $T=L^{*} L$ and $\rho=0$, which yields

$$
A_{\Lambda}=\left|\mu_{u^{\dagger}, u}\right|([0, \Lambda]) \leq\left\|E_{[0, \Lambda]} u^{\dagger}\right\|\|u\| .
$$

If the spectral tail of $u^{\dagger}$ has order $\nu$, we have a constant $C>0$ so that $\left\|E_{[0, \lambda]} u^{\dagger}\right\| \leq C \lambda^{\frac{\nu}{2}}$ and thus

$$
A_{\Lambda} \leq C\|u\| \Lambda^{\frac{\nu}{2}} .
$$

For $B_{\Lambda}$, we also use the inequality (23) with $T=L^{*} L$, and get for arbitrary $\rho \in \mathbb{R}$, the upper bound

$$
B_{\Lambda}=\left|\mu_{u^{\dagger}, u}\right|([\Lambda, \infty)) \leq\left\|\left(L^{*} L\right)^{\frac{\rho}{2}} u\right\|\left(\int_{[\Lambda, \infty)} \frac{1}{\lambda^{\rho}} \mathrm{d} \mu_{u^{\dagger}, u^{\dagger}}(\lambda)\right)^{\frac{1}{2}} .
$$

Choosing now $\rho>\nu$, we can estimate the integral with (24) (using that the measure $\mu_{u^{\dagger}, u^{\dagger}}$ satisfies $\left.\mu_{u^{\dagger}, u^{\dagger}}([0, \lambda])=\left\|E_{[0, \lambda]} u^{\dagger}\right\|^{2} \leq C^{2} \lambda^{\nu}\right)$ and find

$$
B_{\Lambda} \leq \frac{C}{\sqrt{1-\frac{\nu}{\rho}}}\left\|\left(L^{*} L\right)^{\frac{\rho}{2}} u\right\| \Lambda^{\frac{\nu-\rho}{2}} .
$$

Therefore, recalling that $\Lambda$ was chosen so that $A_{\Lambda} \geq \frac{1}{2} A_{\infty}$ and $B_{\Lambda} \geq \frac{1}{2} A_{\infty}$, we have

$$
2\left\langle u^{\dagger}, u\right\rangle \leq 2 A_{\infty} \leq 4 A_{\Lambda}^{1-\frac{\nu}{\rho}} B_{\Lambda}^{\frac{\nu}{\rho}} \leq \frac{4 C}{\left(1-\frac{\nu}{\rho}\right)^{\frac{\nu}{2 \rho}}}\|u\|^{1-\frac{\nu}{\rho}}\left\|\left(L^{*} L\right)^{\frac{\rho}{2}} u\right\|^{\frac{\nu}{\rho}},
$$

which is the condition (ii). 
For the other direction, we remark that from the inequality (20) for some constant $\beta \geq 0$, we find for every $\lambda \geq 0$ that

$$
\left\|E_{[0, \lambda]} u^{\dagger}\right\|^{2}=\left\langle E_{[0, \lambda]} u^{\dagger}, u^{\dagger}\right\rangle \leq \frac{\beta}{2}\left\|\left(L^{*} L\right)^{\frac{\rho}{2}} E_{[0, \lambda]} u^{\dagger}\right\|^{\frac{\nu}{\rho}}\left\|E_{[0, \lambda]} u^{\dagger}\right\|^{1-\frac{\nu}{\rho}} .
$$

Now, since $E$ is the spectral measure of $L^{*} L$, we have that $\left\|\left(L^{*} L\right)^{\frac{\rho}{2}} E_{[0, \lambda]} u^{\dagger}\right\| \leq$ $\lambda^{\frac{\rho}{2}}\left\|E_{[0, \lambda]} u^{\dagger}\right\|$, see for example [2, Chapter X.2.9, Corollary 9], and we therefore obtain that

$$
\left\|E_{[0, \lambda]} u^{\dagger}\right\|^{2} \leq \frac{\beta}{2} \lambda^{\frac{\nu}{2}}\left\|E_{[0, \lambda]} u^{\dagger}\right\|
$$

which concludes the proof.

Remark: In fact, it can be seen from this proof that condition (ii) in Proposition 11 also implies condition (i) in the case $\rho=\nu=2$, which corresponds to the result that the standard source condition for $\nu=2$ yields a noise-free convergence rate of order 1 .

Finally, we can summarize all the statements in an equivalence result between the different source conditions and convergence rates.

Theorem 12. Let $L: U \rightarrow V$ be a bounded linear operator between two real Hilbert spaces $U$ and $V$ and $y \in \mathcal{R}(L)$.

Then, for every $\nu \in(0,2)$, it is equivalent for the problem (1) that

(i) it fulfils the symmetrized variational inequality with parameter $\nu$,

(ii) it has a noise-free convergence rate of order $\frac{\nu}{2}$,

(iii) it has a convergence rate of order $\frac{\nu}{\nu+1}$,

(iv) its minimum-norm solution $u^{\dagger}$ has a spectral tail of order $\nu$,

and if $\nu \in(0,1)$ these are additionally equivalent to

(v) the homogeneous variational inequality with parameter $\nu$ and

(vi) the inhomogeneous variational inequality with parameter $\mu=\frac{2 \nu}{1+\nu}$.

Proof: We already know from Proposition 10 that (ii), (iii), and (iv) are equivalent conditions. Moreover, we know from Lemma 2 that (v) implies (vi), and from Proposition 8 that (vi) implies (ii) and (iii).

Now, Proposition 11 with $\rho=1$ shows that (iv) implies (v), which proves the equivalence of all conditions but (i).

And finally, the equivalence of (i) and (iv) follows directly from Proposition 11 with $\rho=2$.

We briefly comment on the case $\nu=1$. We have already seen in Lemma 2 that in this case the standard source condition, the homogeneous variational inequality and the inhomogeneous variational inequality (all with the parameter 1) are equivalent. Moreover, because of Proposition 8 they also imply all the conditions of Proposition 10. However, the converse is not true. 
Example 13. Let $U$ be a real, separable Hilbert space with orthonormal basis $\left\{\varphi_{n}\right\}_{n \in \mathbb{N}}$. We define the compact linear operator $L: U \rightarrow U$ by $L \varphi_{n}=n^{-\frac{1}{2}} \varphi_{n}$.

Then, for the data

$$
y=\sum_{n \geq 1} n^{-\frac{3}{2}} \varphi_{n} \in \mathcal{R}(L),
$$

the minimum-norm solution $u^{\dagger}$ of problem (1) has spectral tail of order 1, but the problem does not fulfill the inhomogeneous variational inequality with the parameter $\mu=1$.

Proof: We see that the minumum norm solution $u^{\dagger}$ is explicitly given by

$$
u^{\dagger}=\sum_{n \geq 1} n^{-1} \varphi_{n}
$$

and thus has a spectral tail of order 1:

$$
\left\|E_{[0, \lambda]} u^{\dagger}\right\|^{2}=\sum_{n \geq \lambda^{-2}}\left|\left\langle u^{\dagger}, \varphi_{n}\right\rangle\right|^{2}=\sum_{n \geq \lambda^{-2}} n^{-2} \leq C \lambda^{2}
$$

for all $\lambda \in[0,\|L\|]$ for some constant $C>0$, where $A \mapsto E_{A}$ again denotes the spectral measure of $L^{*} L$.

However, for $u_{N}=N^{-1} \sum_{n \leq N} \varphi_{n}$, we find that

$$
\frac{2\left\langle u^{\dagger}, u_{N}\right\rangle}{\left\|L u_{N}\right\|+\left\|u_{N}\right\|^{2}}=\frac{2 N^{-1} H_{N}}{N^{-1} H_{N}^{1 / 2}+N^{-2} \sum_{n \leq N} 1} \geq H_{N}^{1 / 2},
$$

where $H_{N}:=\sum_{n \leq N} n^{-1}$ denotes the $N$-th harmonic number. Because $H_{N} \rightarrow$ $\infty$ as $N \rightarrow \infty$, the inhomogeneous variational inequality with parameter $\mu=1$ cannot be satisfied.

The condition (4) seems to be the natural condition for convergence rates. It is a necessary and sufficient condition for the rate $\mathcal{O}\left(\alpha^{\frac{\nu}{2}}\right)$, while the standard range condition (3) leaves a small gap.

Theorem 12 guarantees that the variational source conditions are optimal conditions for convergence rates.

\section{Acknowledgment}

This work has been supported by the Austrian Science Fund (FWF) within the national research network Geometry + Simulation (project S11704, Variational Methods for Imaging on Manifolds). This research was supported in part by National Science Foundation grant CMG DMS-1025318, and in part by the members of the Geo-Mathematical Imaging Group at Purdue University. 


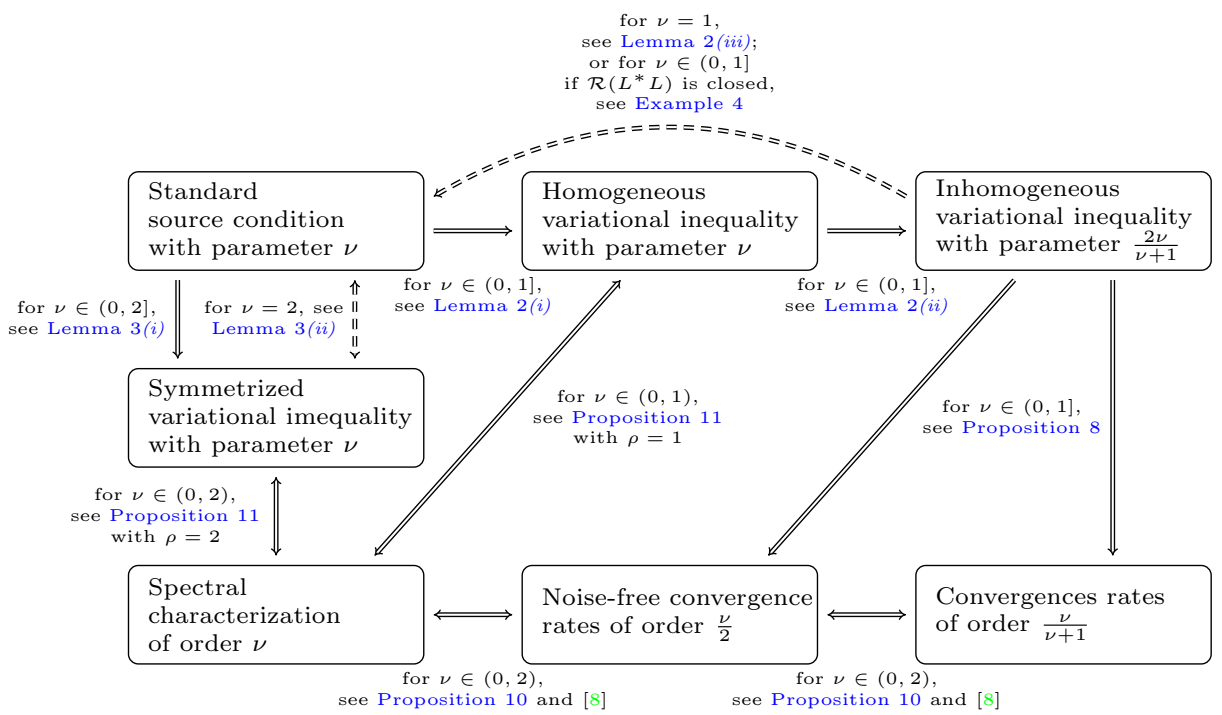

Figure 1: Relation between the different source conditions and the convergence rate results.

\section{Conclusion}

In this paper we have developed a series of novel variational source conditions as alternatives to classical source conditions to prove convergence rates results for Tikhonov regularization in an Hilbert space setting. In many cases the new source conditions provide optimal convergence rates, opposed to the standard source conditions. The interplay between various source conditions and convergence rates is developed in detail and summarized in Table 1. As a side product we could clarify an open question in [10]. An open question is of course how these results can be generalized to non-linear ill-posed problems, to Banach spaces or general topological spaces, and to other regularization methods.

\section{Appendix}

Lemma 14. Let $T: U \rightarrow U$ be a self-adjoint, non-negative definite, bounded linear operator on a real Hilbert space $U, u^{\dagger}, u \in U$, and $\Lambda \geq 0$. We denote with $A \mapsto E_{A}$ the projection-valued spectral measure of $T$ and define for all $v, w \in U$ the signed measure $A \mapsto \mu_{v, w}(A)=\left\langle E_{A} v, w\right\rangle$.

Then, we have for all $0 \leq a \leq b$ and every $\rho \in \mathbb{R}$ that

$$
\left|\mu_{u^{\dagger}, u}\right|([a, b]) \leq\left(\int_{[a, b]} \lambda^{-\rho} \mathrm{d} \mu_{u^{\dagger}, u^{\dagger}}(\lambda)\right)^{\frac{1}{2}}\left(\int_{[a, b]} \lambda^{\rho} \mathrm{d} \mu_{u, u}(\lambda)\right)^{\frac{1}{2}} .
$$


Proof: Using the spectral representation theorem, see for instance [2, Chapter X.5.3, Corollary 4], we may assume that the operator $T$ is a multiplication operator $u \mapsto m u$, where $m \geq 0$ is a bounded measurable function on a measure space $(\Omega, \Sigma, \mu)$ and $U$ is the Lebesgue space $U=L^{2}(\Omega ; \mu)$. In this case, the spectral measure of $T$ is given by $\left\langle E_{A} u^{\dagger}, u\right\rangle=\int_{m^{-1}(A)} u^{\dagger} u \mathrm{~d} \mu$. Therefore, for every $\rho \in \mathbb{R}$, we have the representation

$$
\int_{[a, b]} \lambda^{\rho} \mathrm{d}\left|\mu_{u^{\dagger}, u}\right|(\lambda)=\int_{m^{-1}([a, b])} m^{\rho}\left|u^{\dagger}\right||u| \mathrm{d} \mu .
$$

Thus we can estimate with the Cauchy-Schwarz inequality for arbitrary $\rho \in \mathbb{R}:$

$$
\begin{aligned}
\left|\mu_{u^{\dagger}, u}\right|([a, b]) & =\int_{m^{-1}([a, b])}\left|u^{\dagger}\right||u| \mathrm{d} \mu(\lambda) \\
& \leq\left(\int_{m^{-1}([a, b])} m^{-\rho}\left|u^{\dagger}\right|^{2} \mathrm{~d} \mu\right)^{\frac{1}{2}}\left(\int_{m^{-1}([a, b])} m^{\rho}|u|^{2} \mathrm{~d} \mu\right)^{\frac{1}{2}} \\
& =\left(\int_{[a, b]} \lambda^{-\rho} \mathrm{d} \mu_{u^{\dagger}, u^{\dagger}}(\lambda)\right)^{\frac{1}{2}}\left(\int_{[a, b]} \lambda^{\rho} \mathrm{d} \mu_{u, u}(\lambda)\right)^{\frac{1}{2}} .
\end{aligned}
$$

Lemma 15. Let $\mu$ be a non-negative finite Borel measure on $\mathbb{R}$ with compact support in $[0, \infty)$. Let $0 \leq \nu<\rho$. Suppose that there exists a constant $C>0$ such that $\mu([0, \lambda)) \leq C \lambda^{\nu}$ for all $\lambda \geq 0$. Then

$$
\int_{[\Lambda, \infty)} \lambda^{-\rho} \mathrm{d} \mu(\lambda) \leq C \frac{\rho}{\rho-\nu} \Lambda^{\nu-\rho} \text { for all } \Lambda>0 .
$$

Proof: For $\lambda \geq 0$ define $I(\lambda):=\mu([0, \lambda))$ and $g(\lambda):=\lambda^{-\rho}$. Then we can write the above integral as a Stieltjes integral, and apply integration by parts,

$$
\begin{aligned}
\int_{[\Lambda, \infty)} \lambda^{-\rho} \mathrm{d} \mu(\lambda) & =\int_{\Lambda}^{\infty} g(\lambda) \mathrm{d} I(\lambda) \\
& =\left.g(\lambda) I(\lambda)\right|_{\substack{\lambda=\Lambda \\
\lambda=\Lambda}}-\int_{\Lambda}^{\infty} I(\lambda) \mathrm{d} g(\lambda) .
\end{aligned}
$$

Because $\mu$ is finite and $g(\infty)=0$ it follows that $\left.g(\lambda) I(\lambda)\right|_{\lambda=\Lambda} ^{\lambda=\infty} \leq 0$. Therefore, and taking into account that $g$ is smooth, it follows that

$$
\int_{[\Lambda, \infty)} \lambda^{-\rho} \mathrm{d} \mu(\lambda) \leq-\int_{\Lambda}^{\infty} I(\lambda) \mathrm{d} g(\lambda)=-\int_{\Lambda}^{\infty} I(\lambda) g^{\prime}(\lambda) \mathrm{d} \lambda .
$$

Then we use the assumption $I(\lambda) \leq C \lambda^{\nu}$ and the monotonicity of $g$, to see that

$$
\int_{[\Lambda, \infty)} \lambda^{-\rho} \mathrm{d} \mu(\lambda) \leq-C \int_{\Lambda}^{\infty} \lambda^{\nu} g^{\prime}(\lambda) \mathrm{d} \lambda=C \frac{\rho}{\rho-\nu} \Lambda^{\nu-\rho} .
$$

Thus the assertion is proved. 


\section{References}

[1] J. Cheng and M. Yamamoto. One new strategy for a priori choice of regularizing parameters in Tikhonov's regularization, Inverse Problems, volume 16, L31-L38, 2000.

[2] N. Dunford and J.T. Schwartz. Linear Operators I,II. Wiley, New York, 1963.

[3] H. W. Engl, M. Hanke, and A. Neubauer. Regularization of inverse problems, volume 375 of Mathematics and its Applications. Kluwer Academic Publishers Group, Dordrecht, 1996.

[4] C. W. Groetsch. The Theory of Tikhonov Regularization for Fredholm Equations of the First Kind. Pitman, Boston, 1984.

[5] T. Hein and B. Hofmann. Approximate source conditions for nonlinear illposed problems - chances and limitations. Inverse Problems, 25:035003, 2009.

[6] B. Hofmann, B. Kaltenbacher, C. Pöschl, and O. Scherzer. A convergence rates result for Tikhonov regularization in Banach spaces with non-smooth operators. Inverse Probl., 23(3):987-1010, 2007.

[7] B. Hofmann and M. Yamamoto. On the interplay of source conditions and variational inequalities for nonlinear ill-posed problems. Appl. Anal., 89(11):1705-1727, 2010.

[8] A. Neubauer. On converse and saturation results for Tikhonov regularization of linear ill-posed problems. SIAM J. Numer. Anal., 34:517-527, 1997.

[9] O. Scherzer, M. Grasmair, H. Grossauer, M. Haltmeier, and F. Lenzen. Variational methods in imaging, volume 167 of Applied Mathematical Sciences. Springer, New York, 2009.

[10] T. Schuster, B. Kaltenbacher, B. Hofmann, and K. S. Kazimierski. Regularization methods in Banach spaces, volume 10 of Radon Series on Computational and Applied Mathematics. Walter de Gruyter GmbH \& Co. KG, Berlin, 2012.

[11] http://math.stackexchange.com/questions/853764/ 